\title{
Stress-induced modulation of nociceptive responses in the rat
}

\section{anterior cingulate cortex}

Hiromi Yamashita ${ }^{1,2}$, Jorge L Zeredo, , Kei Kaida?,

Mari Kimoto',4, Izumi Asahina1, Kazuo Toda²

${ }^{1}$ Oral Surgery, 2 Integrative Sensory Physiology, Graduate School of Biomedical

Sciences, Nagasaki University, Nagasaki 852-8588, Japan, 3University of Brasilia, Brasilia 72220-140, Brazil, ${ }^{4}$ Physiological Laboratories, Japan Women's University, Tokyo 112-8681, Japan.

Running title: Stress modulation of anterior cingulate cortex

Correspondence to: Prof. Kazuo Toda, Integrative Sensory Physiology, Graduate

School of Biomedical Sciences, Nagasaki University, Nagasaki 852-8588, Japan, TEL/FAX:+81-95-8197636, Email:k-toda@nagasaki-u.ac.jp 


\section{ABSTRACT}

In the limbic system, the anterior cingulate cortex (ACCX) is one of the key areas involved in the close association between pain and emotion. However, neuronal changes in ACCX nociceptive responses after stress conditioning have not yet been quantitatively investigated. We investigated the modulation of nociceptive responses in the ACCX neurons following restraint stress in rats. The present study demonstrated that stress-conditioning enhanced excitatory nociceptive responses in the ACCX following tail stimuli in the mid-term (7 days). Short-term (3 days) and long-term (21 days) of stress conditioning did not affect these responses significantly. Nociceptive responses evoked by other sites of the body (nose, back and four paws) stimulation were not changed by stress-conditioning, indicating that neural information from the tail is important for emotional system modulation. It is suggested that the emotional/affective part of the pain sensation is strongly modified by stress through neuroplasticity in the ACCX.

\section{(Keywords)}

Anterior cingulate cortex, limbic system, stress, emotion, pain 


\section{Introduction}

Pain is an emotional experience as well as a sensation. Recently, there has been growing interest in the relation between pain and emotion (Vogt, 2005). It has been reported that the limbic system has a critical role in emotional processing mechanisms for evoking unpleasant feelings, including fear, anxiety or pity, in individuals in a state of pain (Rainville, 2002). In the limbic system, the anterior cingulate cortex $(\mathrm{ACCX})$ is one of the key sites to provide a close relationship between pain and emotion (Bantick, et al., 2002; Bush, et al., 2000; Hutchison, et al., 1999; Toda, 1992; Vogt, 2005).

Anatomically, the ACCX receives its afferent axons primarily from the medial part of the thalamic nuclei, which is considered to be an emotional relay site in the pain pathway (Shyu, et al., 2010; Sikes, et al., 2008; Treede, et al., 1999). Functional studies have shown that lesion of the ACCX could alleviate the emotional reaction to painful stimuli, while the ability to localize such stimuli remained unaffected (Cole, et al., 2006; Devinsky, et al., 1995; Rainville, 2002). Behavioral studies in monkeys showed that stimulation of the ACCX can produce shrill vocalization, which is presumed to be associated with emotion 
evoked by noxious stimulation (Baleydier\& Mauguiere, 1980; Vogt, 2005). In addition, behavioral studies have shown convincing evidences that the ACCX is strongly involved in the sensory-affective processing of nociception. The ACCX mediates the affective response of tonic pain in the hot-plate test, the formalin test, and the formalin-induced conditioned place avoidance test, all in rodents (Johansen, et al., 2001; Sikes\& Vogt, 1992). Likewise, in humans, several functional brain imaging studies of nociceptive responses have shown that the ACCX mediates the affective response to acute, noxious heat stimulation (Casey, 1999; Hutchison, et al., 1999; Villemure\& Bushnell, 2009).

On the other hand, stress often modifies nociceptive sensitivities, as it activates multiple neuronal and endocrine systems that modify pain sensation. Until now, two opposite results have been obtained in relation to the effects of stress on pain (Butler\& Finn, 2009; Yilmaz, et al., 2010). For example, acute stressors reduce pain, a phenomenon called stress-induced antinociception (Yamaguchi, et al., 2003). After gravity stress-conditioning, the threshold value of the withdrawal reflex elicited by mechanical stimulation was increased (Kawauchi, et al., 2004). By contrast, chronic stress facilitates pain transmission in animal 
models (Imbe, et al., 2004; Imbe, et al., 2010) and in patients (Karp, et al., 2008). Thus, stress-induced hyperalgesia is observed in animals and also in humans. It is thought that the magnitude, duration, and nature of the stressor are critical factors for producing stress-induced hyperalgesia (Gibbons, et al., 2012; Imbe, et al., 2006).

In summary, neuronal systems in the limbic system are strongly involved in both stress and pain. In particular, the ACCX may be the key brain site in the stress-pain interaction. However, neuronal changes in ACCX nociceptive responses after stress conditioning have not yet been quantitatively investigated. Therefore, in the present study, we investigated modulation of nociceptive responses in the ACCX neurons following stress-conditioning in rats. 


\section{Methods}

\subsection{General}

The methods described here follow the ethical guidelines and received approval by the Animal Welfare Committee of Nagasaki University. Twenty Wistar albino rats (male, initial body weight of about $160 \mathrm{~g}$ ) were used. Animals were kept in a temperature-controlled room $\left(24 \pm 1^{\circ} \mathrm{C}\right)$ under a $12 \mathrm{~h}: 12 \mathrm{~h}$ light/dark cycle (lights on from 12:00 to $24: 00)$. The animals were adapted to this cycle for one week before stress-conditioning. Rats were randomly assigned to four groups of equal size ( $n=5$, each): wire mesh restraint for 3,7 , and 21 days or a non-restraint control group. All rats were permitted ad lib access to water and standard chow, except during stress-conditioning.

\subsection{Stress-conditioning}

Rats were exposed to restraining stress by placing them individually in wire mesh restrainers for six hours per day (start time: 13:00) during 3, 7 or 21 days. The wire mesh restrainer had a stainless wire mesh attached to a wooden base, and measured $22 \mathrm{~cm}$ in length and $26 \mathrm{~cm}$ in circumference. 


\subsection{Pinch stimulation and electrophysiological recordings}

As shown in Fig.1, noxious pinch stimulation was applied to eight sites (nose, left and right forepaws, upper and lower back, left and right hindpaws, and tail) on the rat's body surface for $5 \mathrm{~s}$ using a blunt serrated forceps. First, we tested response properties to nose stimulation and confirmed the receptive field from the nose. After that, we stimulated randomly the other 7 sites.

Force of pinch was quantitatively adjusted to $1.5 \mathrm{~N}$ by orthodontic elastic bands (L16, TOMY, Tokyo, Japan). When this pinch stimulation was applied to experimenter's hand skin, clear pain sensation was evoked. ACCX neuronal responses were classified according to the number of spikes occurring $5 \mathrm{~s}$ before and $5 \mathrm{~s}$ after pinch stimulation. We did not analyze spike responses during the $5 \mathrm{~s}$ of pinch stimulation, because during that time biological spike responses were often contaminated with large electrical artifacts. Each site was tested 2-3 times in each rat.

In stress groups, immediately after the stop mesh restraint, the animals were anesthetized with thiamylal sodium (Isozol ${ }^{\mathrm{TM}}$, Nippon Iko Pharmacy, Toyama, Japan) initially given $80 \mathrm{mg} / \mathrm{kg}$ intraperitoneally and additionally given $10 \mathrm{mg} / \mathrm{kg}$ 
intravenously every $20 \mathrm{~min}$, through a cannula inserted into the femoral vein. Respiratory conditions, blood pressure, and rectal temperature were continuously monitored and kept within normal physiological levels. Control group received no restraint, but recording procedure was the same as in the stress groups. First, under surgical anesthesia (confirmed by lack of nociceptive withdrawal reflexes), the skull over the medial part of the cerebral cortex was removed bilaterally, and the exposed brain surface was covered with liquid paraffin. Then, a tungsten microelectrode (FDH 25-10, tip impedance 10-12 M $\Omega$, Brunswick, USA) was gently lowered into the ACCX using a micromanipulator (Narishige, Tokyo, Japan). The stereotaxic coordinates for the ACCX were antero-posterior: $0.0-3.0 \mathrm{~mm}$ rostral to bregma, lateral: $0.0-1.2 \mathrm{~mm}$ from the midline, and depth: 1.0-1.4 mm ventral to cortical surface (Swanson, 1992). Single unit recordings were obtained from left ACCX neurons. Neuronal recordings were obtained under constant light-surgical anesthesia, where withdrawal reflexes were mostly suppressed. Electrical signals were amplified by a biological amplifier (DAM 80, WPI Instruments, Sarasota, USA) and continuously displayed and recorded on a Windows PC through the 1401 interface (CED, Cambridge, U.K.). Criteria of single unit were constant amplitude 
and width of spikes, which was checked by discriminative program of Spike2 software (Version 4, CED, Cambridge, U.K.).

The sites of recording in the ACCX were marked by electrical lesions (2-mA DC current passing for $2 \mathrm{~min}$ ) for histological identification. At the end of the experiment, the rats were killed by an overdose of thiamylal sodium.

\subsection{Statistics}

The ANOVA test (post hoc Bonferroni) was used to determine the statistical significance of the data. Changes in pinch-evoked spike number before and after 5s-pinch stimuli were compared among control (without stress conditioning), 3-, 7- and 21-day stress-conditioned rats. Statistical significance was set at the $5 \%$ level. The software Statview version 5.0 (SAS Institute, Cary, NC, U.S.A.) aided in statistical analysis.

\section{Results}

Total of 57 ACCX neurons were recorded in the present study. Almost all 
neurons had large receptive fields from the entire body. A small number of neurons had no receptive field from upper back $(n=2)$, left forepaw $(n=2)$, right forepaw $(n=4)$, left hindpaw $(n=1)$, right hindpaw $(n=1)$, lower back $(n=4)$ and tail $(n=3)$. In both control and stress-conditioned rats, excitatory (TYPE E) and inhibitory (TYPE I) changes were observed in the ACCX neurons after pinch stimulation. A small number of units were not affected by pinch stimulation (TYPE O). Typical examples of the TYPE E and TYPE I unit responses evoked by tail stimulation after 7 day-stress conditioning are shown in Fig. 2 .

TYPE E responses were not significantly different between control and stress groups when pinch stimulation was applied to any of the stimulation sites, except for the tail (Figs. 3 and 4). In TYPE E responses evoked by tail-pinch stimuli, there was a statistically significant difference between groups as determined by one-way ANOVA $(F(3,26)=4.875, p=.008)$. A Bonferroni post-hoc test revealed that excitatory responses were significantly enhanced after 7 days of stress-conditioning $(23.5 \pm 13.6 \mathrm{~Hz}$ increase, $p=.002)$ as compared with control (6.5 $\pm 1.51 \mathrm{~Hz}$ increase). Statistically significant difference was also found between $3(10.2 \pm 9.0 \mathrm{~Hz}$ increase $)$ and 7 days $(23.5 \pm 13.6 \mathrm{~Hz}$ increase, $p=.007)$ 
of stress conditioning. However, after 3 or 21 days of stress conditioning, excitatory responses were not significantly changed as compared to control during tail stimulation (Fig. 4). There was a tendency for a higher firing frequency during right hindpaw stimulation after 7 days of stress conditioning in fashion similar to that of tail stimulation, but the differences were not statistically significant. TYPE I responses of ACCX neurons were not affected by stress-conditioning in any of the 8 stimulation sites (Figs. 3 and 4).

In the present study, some units changed their response type depending on the stimulation site. For example, the same ACCX unit that displayed a TYPE E response to tail stimulation, displayed a TYPE I response to nose stimulation. These changing patterns were not studied further in the present study. ACCX neuronal recordings were obtained from left hemisphere (Fig.5), but systematic differences in response patterns between ipsi- and contra-lateral stimulation sites could not be detected.

Recording sites in the ACCX are shown in Fig. 5. In this figure, response pattern after tail stimulation is summarized in control, 3-, 7-, 21-day stress-conditioning 
groups. There were no tendencies in type of response in relation to the respective location of the recording site.

\section{Discussion}

The present study demonstrated that stress-conditioning enhanced excitatory nociceptive responses in the ACCX following tail stimuli in a "middle stage" of stress conditioning ( 7 days conditioning). Neither short term stress ( 3 day conditioning) nor long-term stress (21 day conditioning) affected these responses. A similar tendency was observed in the right hindpaw, but without statistical significance. This observation could not be attributed to lateralization, since similar results were obtained from the neuronal responses by stimulation of ipsi- and contra-lateral paws. None of the other stimulation sites tested in this study had their evoked responses in the ACCX changed by stress-conditioning. It is not clear that there is a particular significance for the neural information from the tail for anterior cingulate processing; however, the present study, at least under our experimental conditions, suggests that afferent inputs from the rat tail are important for the emotional system. 
A number of previous studies suggest that the ACCX, which is thought to be one of the higher centers for sensory-emotional integration, is involved in the modulation of pain sensation in humans or noxious responses animals (Rainville, 2002; Toda, 1992; Yamamura, et al., 1996). It has been reported that cingulotomy can diminish affective responses to painful stimuli in humans (Hassenbusch, et al., 1990; Wilkinson, 2000; Yen, et al., 2005). Behavioral studies showed that stimulation of the ACCX could produce shrill vocalization, which might be associated with escape responses in monkeys (Devinsky, et al., 1995; Kirzinger\& Jurgens, 1982). These studies strongly support the idea that the ACCX has an important functional role in processing of pain sensation in relation to emotional factors.

It has been recognized that stress, especially chronic stress, has a great negative effect on the emotional, autonomic, and humoral systems (Lumley, et al., 2011; Ordaz\& Luna, 2012). In the emotional system, the ACCX is the key brain site to modulate negative emotions such as fear, anxiety, and sorrow, but also happiness (Etkin, et al., 2011). Therefore, it is reasonable that stress may modulate the ACCX neuronal activities in addition to behavioral responses. 
In the present study, changes in noxious responses were investigated after stress conditioning. Our present study is the first report to investigate single neuronal activities elicited by noxious stimulation in the ACCX after stress. One important observation is that the excitatory responses of ACCX neurons, but not inhibitory responses, were modulated after stress conditioning. This modulation was significant in the mid-term (7 days) of stress conditioning.

As reported by Yamamura et al. (1996), in the rat ACCX both excitatory and inhibitory responses can be evoked by noxious stimulation. Excitatory and inhibitory responses are also observed in ACCX neurons with descending projection to the PAG after electroacupuncture, a potentially stressful form of somatic stimulation (Hirano, et al., 2008). Therefore, it is apparent that somatosensory stimulation evokes responses in the ACCX in a bimodal fashion (Calejesan, et al., 2000; Vaccarino\& Chorney, 1994).

The reason why only excitatory nociceptive responses were changed by stress conditioning is difficult to discuss; however, we may speculate that this 
phenomenon has a critical role in the enhancement of the emotional component of nociceptive responses. The fact that the maximum ratio of increase in excitatory responses was observed at 7 days of stress-conditioning may support this hypothesis. Response changes in ACCX neurons were observed at 7 days of stress conditioning, but not at 3 and 21 days as compared to the control. After 3 days of stress conditioning, it is presumed that the summation of the stress stimuli was insufficient to induce a functional change in the ACCX neurons. On the other hand, ACCX neurons may have already adapted after after 21 days of stress conditioning. Therefore, there may be a critical time for stress-induced neuronal changes to take place in the ACCX specifically, or in the limbic system as a whole. In the autonomic system, changes in ileal movements occur 1 day after gravity-stress conditioning (Kimoto, et al., 2012). In a motivated regulatory behavior such as the feeding behavior, changes are observed 14-18 days after gravity stress-conditioning (Kimoto, 2011). These results suggest a discrepancy in the timing of stress-induced changes in the CNS. Changes can more readily observed in the autonomic system, than in the limbic system, and finally in behavior. Therefore, it is suggested that a certain critical time may be needed for neuronal plasticity to occur in the ACCX. 
In conclusion, stress-enhanced excitatory nociceptive responses in the ACCX were observed in a mid-term stress conditioning. Because the ACCX is one of the emotional centers in the limbic system, it is suggested that emotional/affective component of the pain sensation may be strongly modified by stress through functional plasticity of ACCX neurons.

\section{Acknowledgements}

This study was supported by JSPS (grant no. 24590727) to KT. Part of this study was presented at the FEPS meeting (Santiago de Compostela, Spain, September 8-11, 2012). 


\section{REFERENCES}

Baleydier, C. \& Mauguiere, F. (1980) The duality of the cingulate gyrus in monkey. Neuroanatomical study and functional hypothesis. Brain, 103, 525-54.

Bantick, S. J., Wise, R. G., Ploghaus, A., Clare, S., Smith, S. M. \& Tracey, I. (2002) Imaging how attention modulates pain in humans using functional MRI. Brain, 125, 310-9.

Bush, G., Luu, P. \& Posner, M. I. (2000) Cognitive and emotional influences in anterior cingulate cortex. Trends Cogn Sci, 4, 215-222.

Butler, R. K. \& Finn, D. P. (2009) Stress-induced analgesia. Prog Neurobiol, 88, 184-202.

Calejesan, A. A., Kim, S. J. \& Zhuo, M. (2000) Descending facilitatory modulation of a behavioral nociceptive response by stimulation in the adult rat anterior cingulate cortex. Eur J Pain, 4, 83-96.

Casey, K. L. (1999) Forebrain mechanisms of nociception and pain: analysis through imaging. Proc Natl Acad Sci US A, 96, 7668-74.

Cole, L. J., Farrell, M. J., Duff, E. P., Barber, J. B., Egan, G. F. \& Gibson, S. J. (2006) Pain sensitivity and fMRI pain-related brain activity in Alzheimer's disease. Brain, 129, 2957-65.

Devinsky, O., Morrell, M. J. \& Vogt, B. A. (1995) Contributions of anterior cingulate cortex to behaviour. Brain, 118 ( Pt 1), 279-306.

Etkin, A., Egner, T. \& Kalisch, R. (2011) Emotional processing in anterior cingulate and medial prefrontal cortex. Trends Cogn Sci, 15, 85-93.

Gibbons, C. H., Adler, G. K., Bonyhay, I. \& Freeman, R. (2012) Experimental hypoglycemia is a human model of stress-induced hyperalgesia. Pain, 153 2204-9.

Hassenbusch, S. J., Pillay, P. K. \& Barnett, G. H. (1990) Radiofrequency cingulotomy for intractable cancer pain using stereotaxis guided by magnetic resonance imaging. Neurosurgery, 27, 220-3. 
Hirano, T., Zeredo, J. L., Kimoto, M., Moritaka, K., Nasution, F. H. \& Toda, K. (2008) Disinhibitory involvement of the anterior cingulate cortex in the descending antinociceptive effect induced by electroacupuncture stimulation in rats. Am J Chin Med, 36, 569-77.

Hutchison, W. D., Davis, K. D., Lozano, A. M., Tasker, R. R. \& Dostrovsky, J. O. (1999) Pain-related neurons in the human cingulate cortex. Nat Neurosci, 2, 403-5.

Imbe, H., Iwai-Liao, Y. \& Senba, E. (2006) Stress-induced hyperalgesia: animal models and putative mechanisms. Front Biosci, 11, 2179-92.

Imbe, H., Murakami, S., Okamoto, K., Iwai-Liao, Y. \& Senba, E. (2004) The effects of acute and chronic restraint stress on activation of ERK in the rostral ventromedial medulla and locus coeruleus. Pain, 112, 361-71.

Imbe, H., Okamoto, K., Donishi, T., Senba, E. \& Kimura, A. (2010) Involvement of descending facilitation from the rostral ventromedial medulla in the enhancement of formalin-evoked nocifensive behavior following repeated forced swim stress. Brain Res, 1329, 103-12.

Johansen, J. P., Fields, H. L. \& Manning, B. H. (2001) The affective component of pain in rodents: direct evidence for a contribution of the anterior cingulate cortex. Proc Natl Acad Sci U S A, 98, 8077-82.

Karp, J. F., Shega, J. W., Morone, N. E. \& Weiner, D. K. (2008) Advances in understanding the mechanisms and management of persistent pain in older adults. Br J Anaesth, 101, 111-20.

Kawauchi, Y., Zeredo, J. L., Takeuchi, Y., Kimoto, M., Kumei, Y., Makita, K. \& Toda, K. (2004) Nociceptive threshold can be changed by gravity stimulation in rats. Stress and Health, 20, 45-50.

Kimoto, M. (2011) [Effect of buprenorphine on postoperative analgesia following remifentanil-based anesthesia]. Masui, 60, 656-60.

Kimoto, M., Zeredo, J. L. \& Toda, K. (2012) Hypergravity conditioning on ileal movements in rats. Aviat Space Environ Med, 83, 483-7.

Kirzinger, A. \& Jurgens, U. (1982) Cortical lesion effects and vocalization in the 
squirrel monkey. Brain Res, 233, 299-315.

Lumley, M. A., Cohen, J. L., Borszcz, G. S., Cano, A., Radcliffe, A. M., Porter, L. S., Schubiner, H. \& Keefe, F. J. (2011) Pain and emotion: a biopsychosocial review of recent research. J Clin Psychol, 67, 942-68.

Ordaz, S. \& Luna, B. (2012) Sex differences in physiological reactivity to acute psychosocial stress in adolescence. Psychoneuroendocrinology, 37, 1135-57.

Rainville, P. (2002) Brain mechanisms of pain affect and pain modulation. Curr Opin Neurobiol, 12, 195-204.

Shyu, B. C., Sikes, R. W., Vogt, L. J. \& Vogt, B. A. (2010) Nociceptive processing by anterior cingulate pyramidal neurons. J Neurophysiol, 103, 3287-301.

Sikes, R. W. \& Vogt, B. A. (1992) Nociceptive neurons in area 24 of rabbit cingulate cortex. J Neurophysiol, 68, 1720-32.

Sikes, R. W., Vogt, L. J. \& Vogt, B. A. (2008) Distribution and properties of visceral nociceptive neurons in rabbit cingulate cortex. Pain, 135, 160-74.

Swanson, L. W. (1992) Brain Maps: Structure of the Rat Brain, Elsevier, Amsterdam.

Toda, K. (1992) Anterior cingulate-induced inhibition of activities of descending periaqueductal gray matter neurons in rats. Pain Res, 7, 71-79.

Treede, R. D., Kenshalo, D. R., Gracely, R. H. \& Jones, A. K. (1999) The cortical representation of pain. Pain, 79, 105-11.

Vaccarino, A. L. \& Chorney, D. A. (1994) Descending modulation of central neural plasticity in the formalin pain test. Brain Res, 666, 104-8.

Villemure, C. \& Bushnell, M. C. (2009) Mood influences supraspinal pain processing separately from attention. J Neurosci, 29, 705-15.

Vogt, B. A. (2005) Pain and emotion interactions in subregions of the cingulate gyrus. Nat Rev Neurosci, 6, 533-44.

Wilkinson, H. A. (2000) Bilateral anterior cingulotomy for chronic noncancer pain. 
Neurosurgery, 46, 1535-6.

Yamaguchi, K., Toda, K. \& Hayashi, Y. (2003) Effects of stressful training on human pain threshold. Stress and Health, 19, 9-15.

Yamamura, H., Iwata, K., Tsuboi, Y., Toda, K., Kitajima, K., Shimizu, N., Nomura, H., Hibiya, J., Fujita, S. \& Sumino, R. (1996) Morphological and electrophysiological properties of ACCx nociceptive neurons in rats. Brain Res, 735, 83-92.

Yen, C. P., Kung, S. S., Su, Y. F., Lin, W. C., Howng, S. L. \& Kwan, A. L. (2005) Stereotactic bilateral anterior cingulotomy for intractable pain. J Clin Neurosci, 12, 886-90.

Yilmaz, P., Diers, M., Diener, S., Rance, M., Wessa, M. \& Flor, H. (2010) Brain correlates of stress-induced analgesia. Pain, 151, 522-9. 


\section{Figure Legends}

Fig. 1. Stimulation sites for pinch. 1: nose, 2: upper back, 3: left and 4: right forepaws, 5: left ,and 6: right hindpaws, 7: lower back, and 8: tail. Nociceptive responses in ACCX neurons were recorded after stimulation of these sites.

Fig.2. Typical examples of TYPE E and TYPE I neuronal responses in the ACCX elicited by tail stimulation a in 7-day stress-conditioned rat. Upper traces: activity before 5-s tail-pinch; Lower traces: activity after 5-s tail-pinch.

Dotted lines: $5 \mathrm{~s}$ immediately before and immediately after tail-pinch. Raw traces during pinch stimulation are not shown because of large electrical artifacts. Calibrations: $1 \mathrm{~s}$ (horizontal) and $0.1 \mathrm{mV}$ (vertical). 
Fig.3. Difference in firing frequency of ACCX nociceptive responses (afterbefore stimulation) according to the stimulation site in Control, 3 days, 7 days, and 21 days of stress-conditioning.

Fig. 4. (Continued from Fig. 3.) Asterisks indicate statistically significant difference (5\% level) in ANOVA followed by Bonferroni post-hoc test.

Fig. 5. Coronal brain sections showing the recording sites of TYPE E (red, $n=30$ ), TYPE I (blue, $n=19$ ) and TYPE O (open, $n=5$ ) neuronal responses to tail-pinch stimulation. Dots are superimposed from histological sections onto plates $+1.7,+1.0$, and $+0.2 \mathrm{~mm}$ rostral to bregma of Swanson's rat brain atlas. 
Figure 1

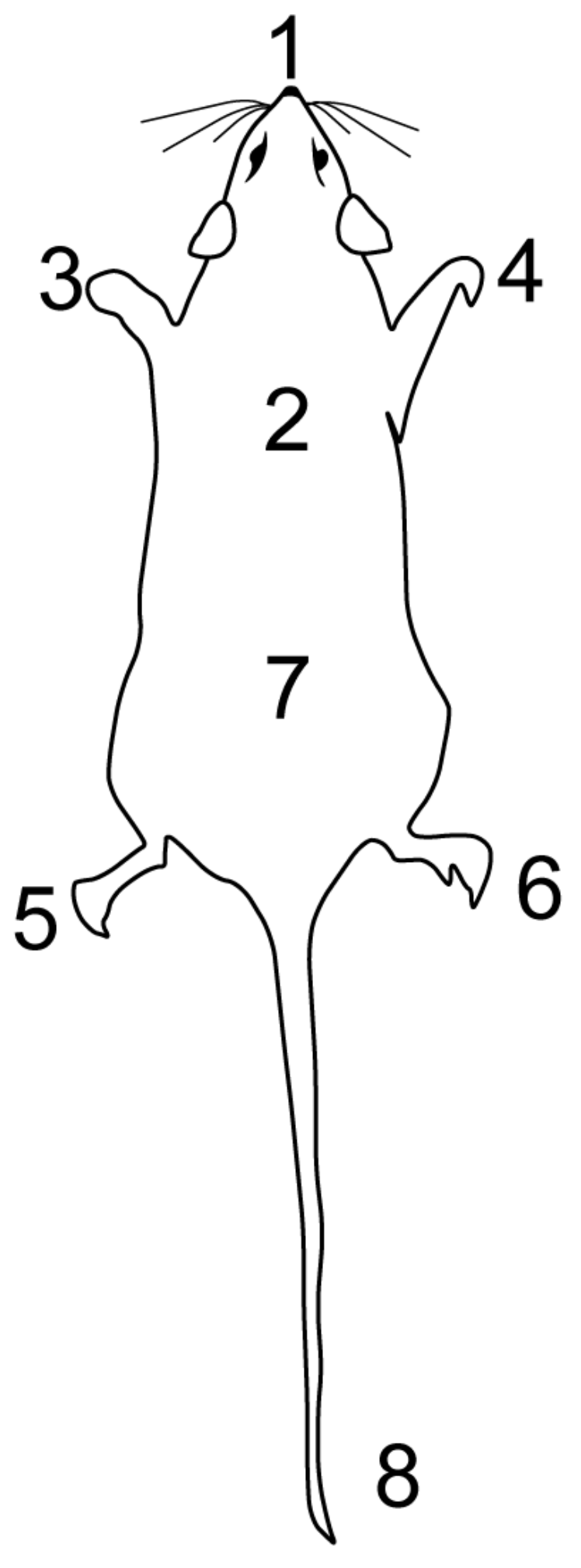

Page $\mathbf{2 3}$ of $\mathbf{2 7}$ 
Figure 2

\section{TYPE E}

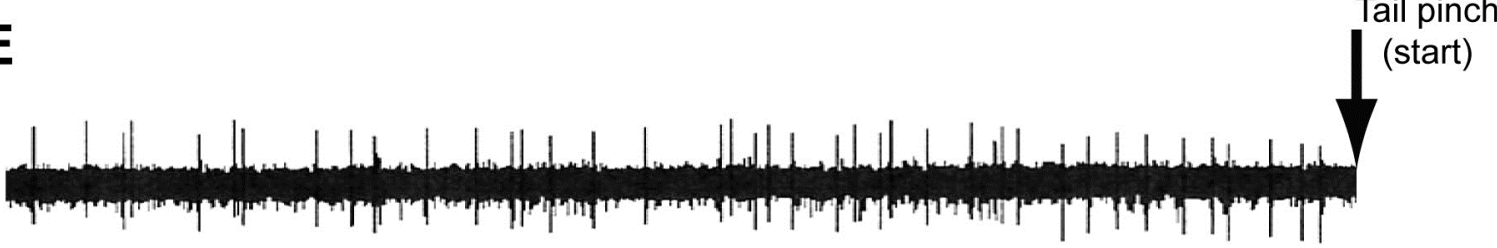

Tail pinch

(end)

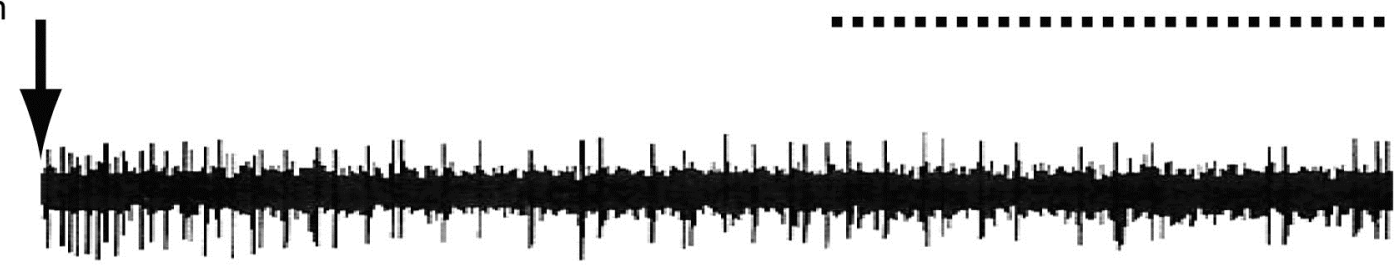

TYPE I

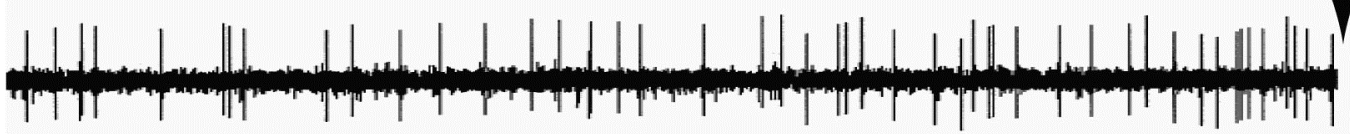

Tail pinch (end)

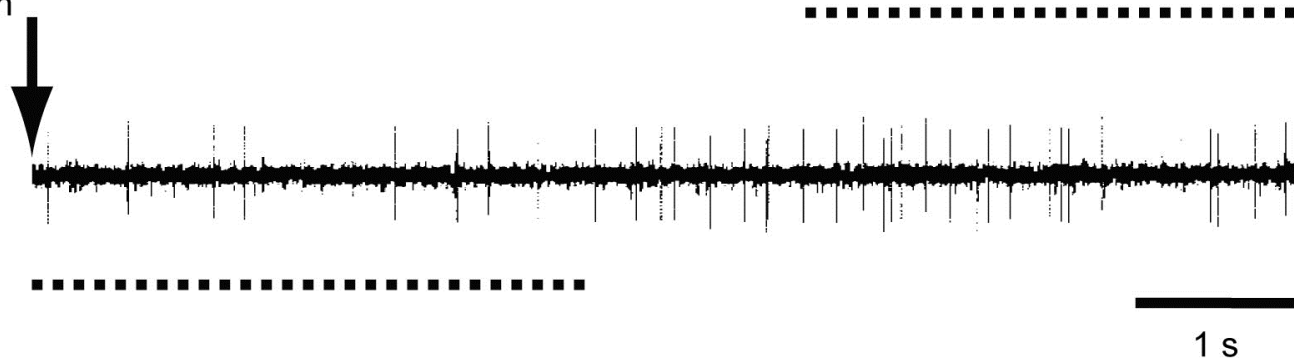


Figure 3

\section{1: Nose}
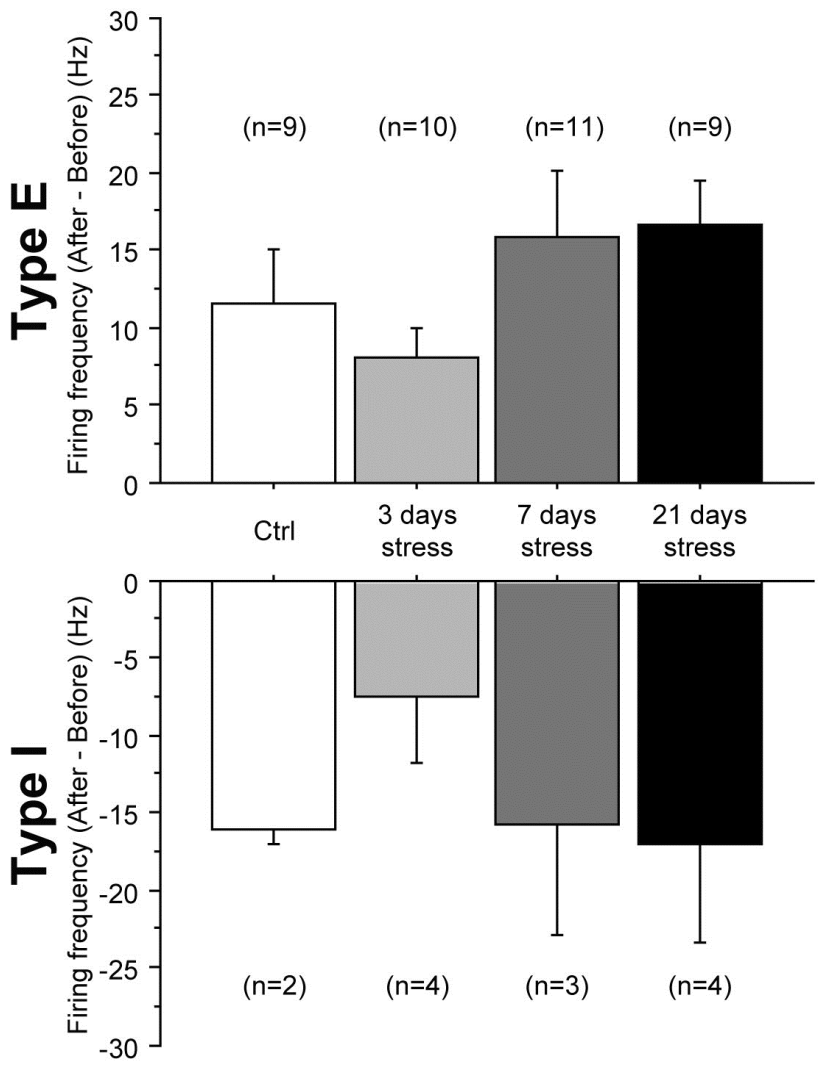

\section{3: Left forepaw}
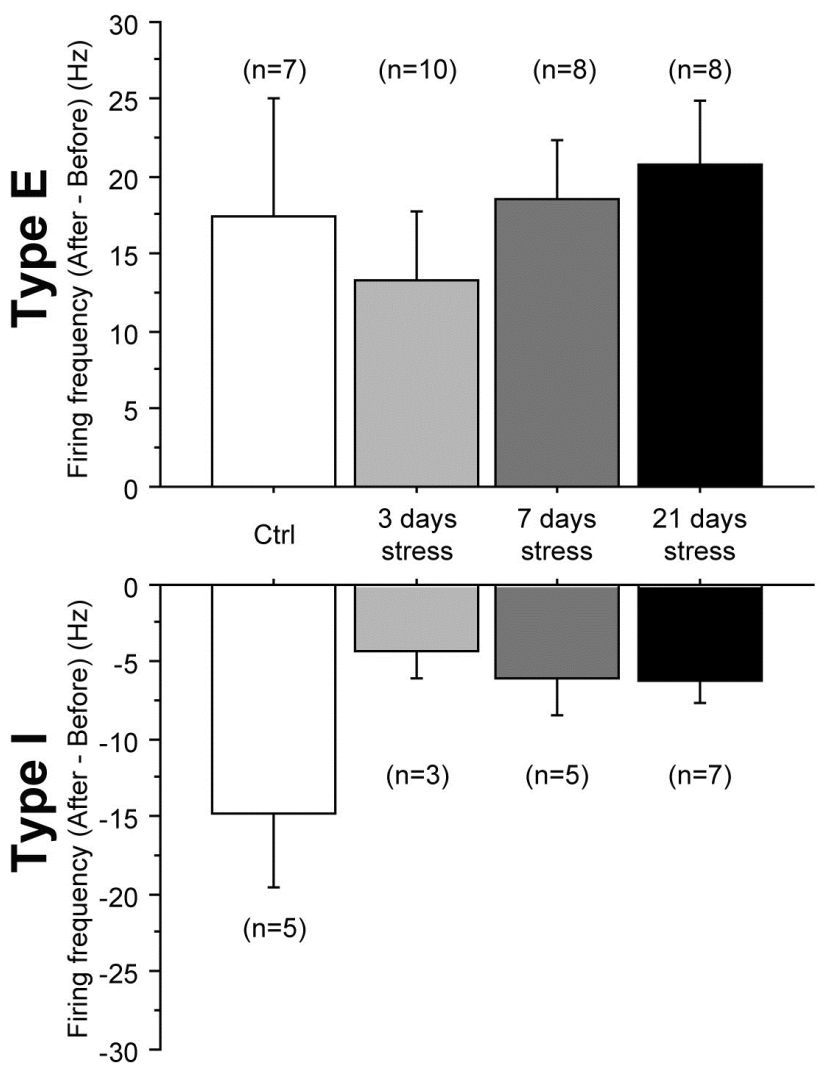

\section{2: Upper back}
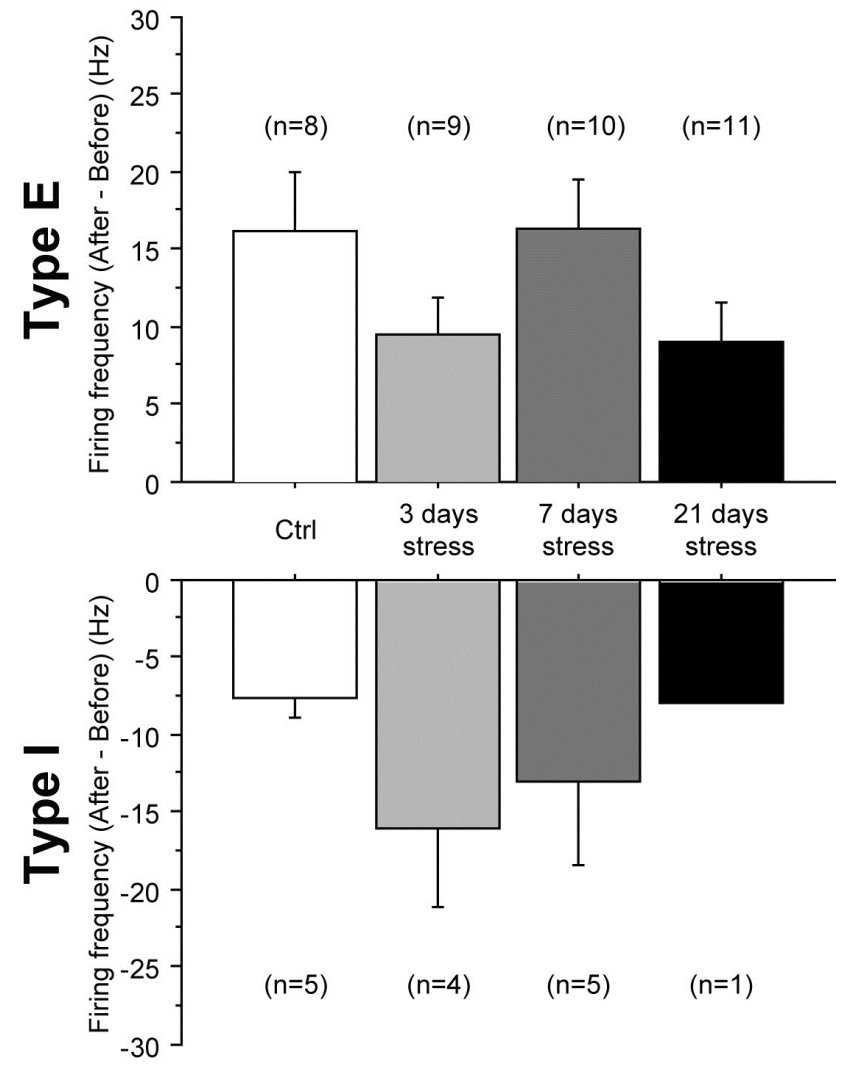

\section{4: Right forepaw}
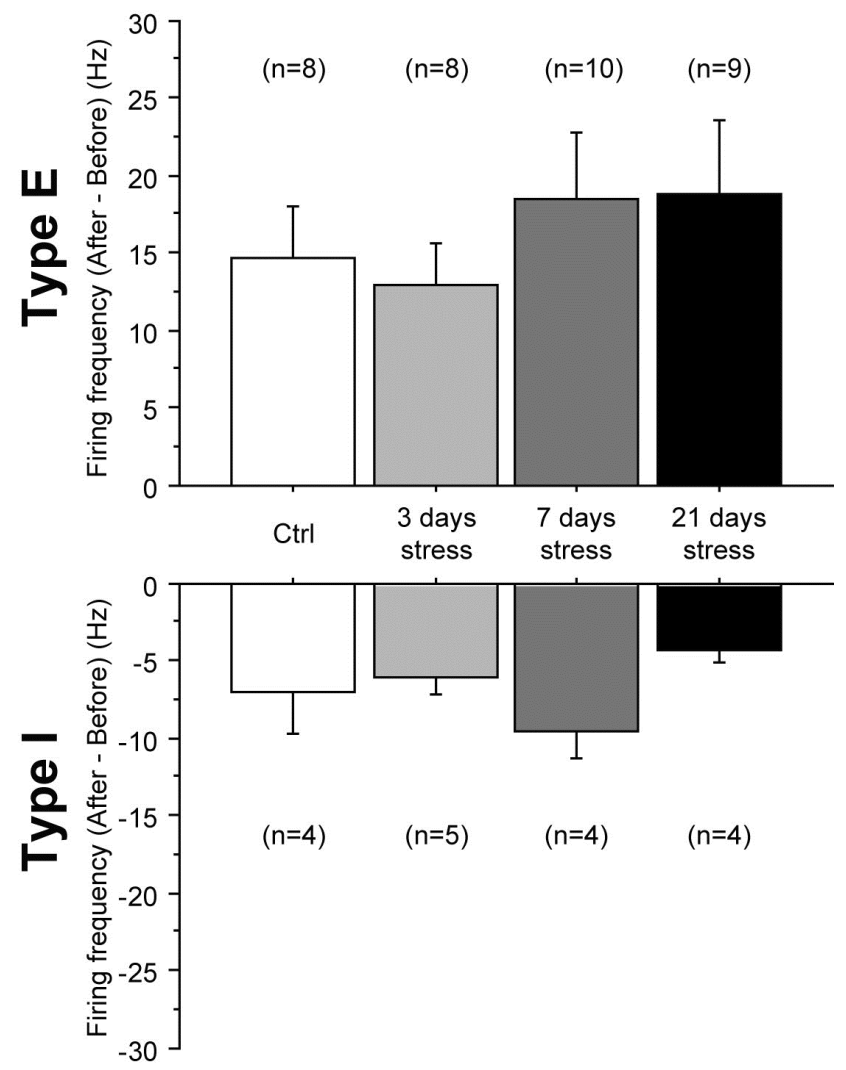
Figure 4

\section{5: Left hindpaw}
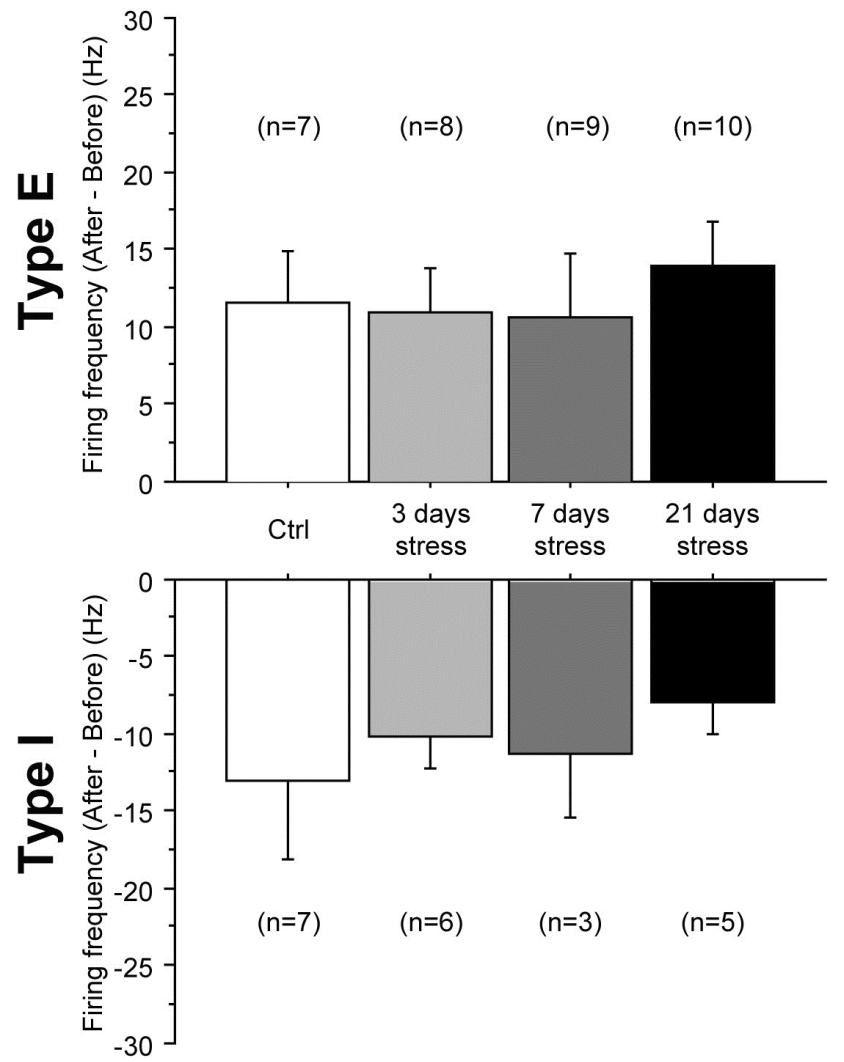

7: Lower back
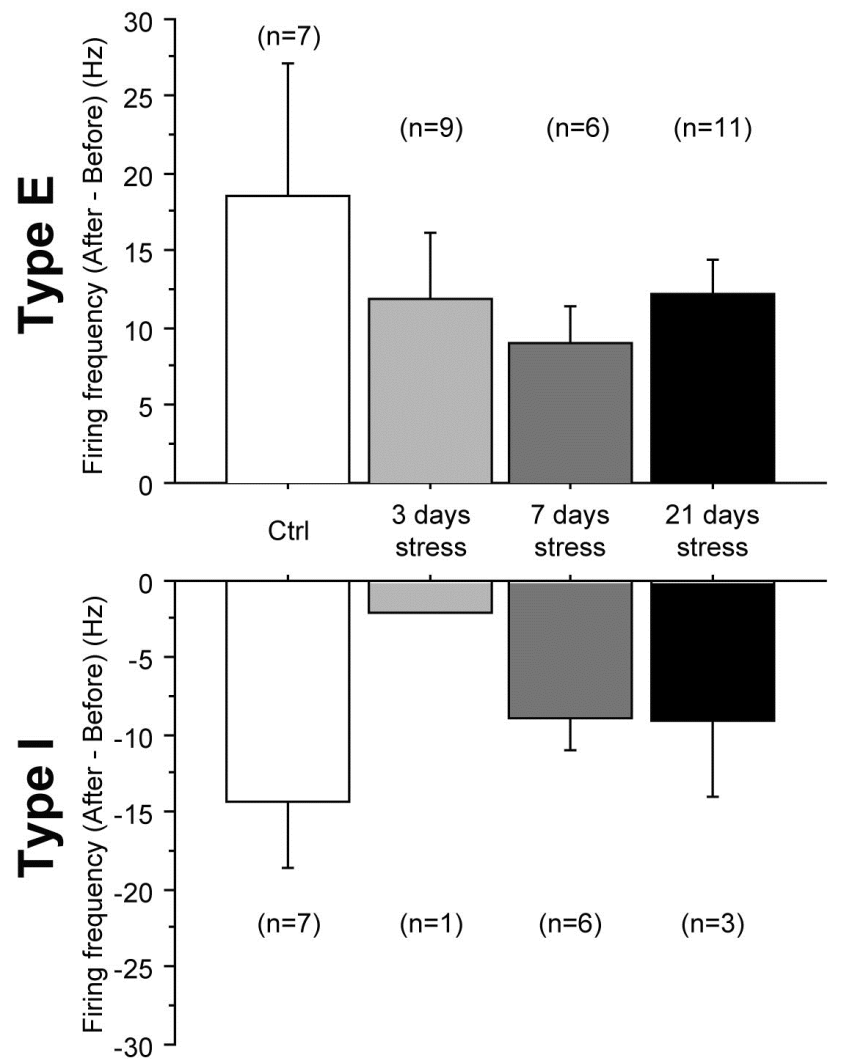

\section{6: Right hindpaw}
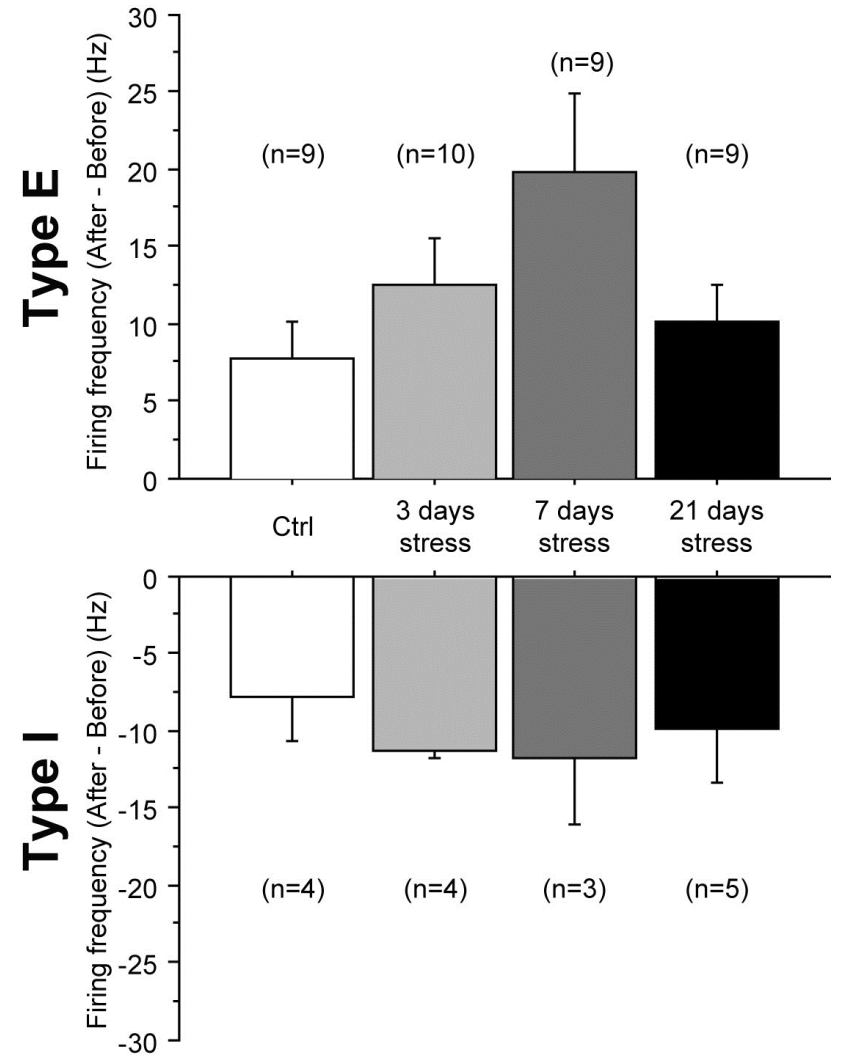

8: Tail
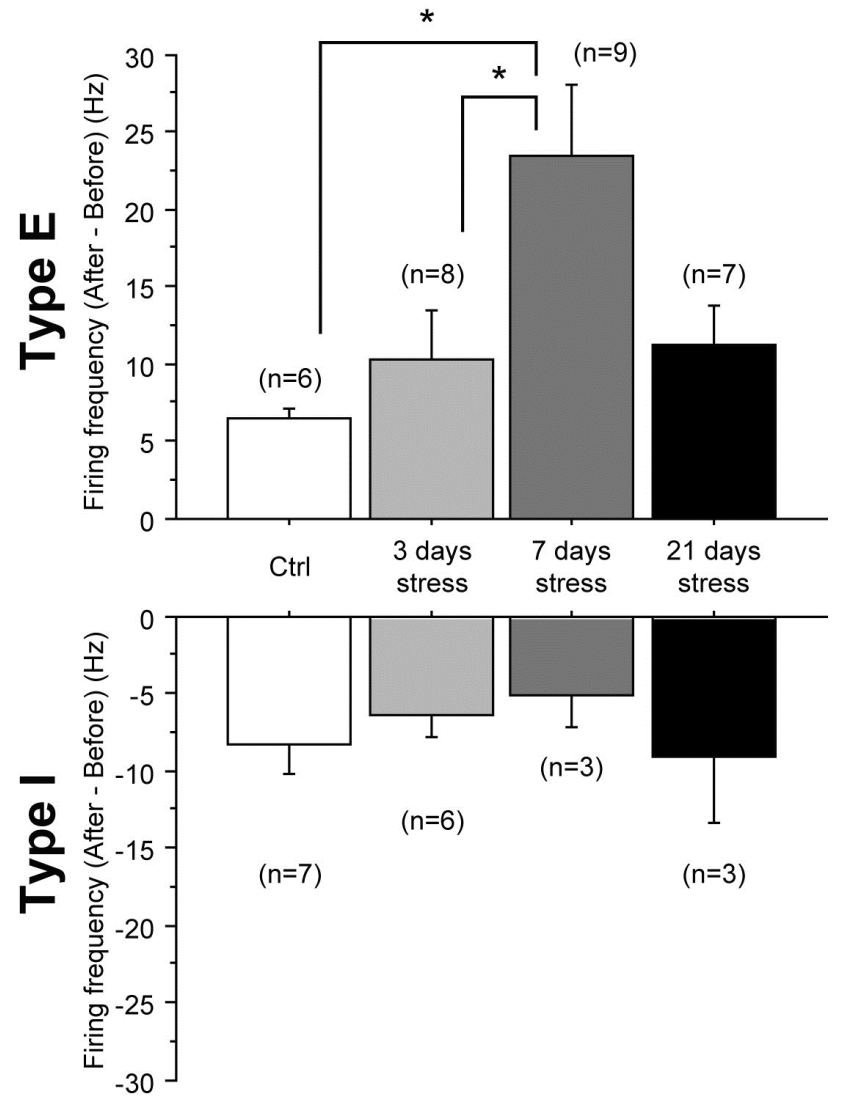
Figure 5

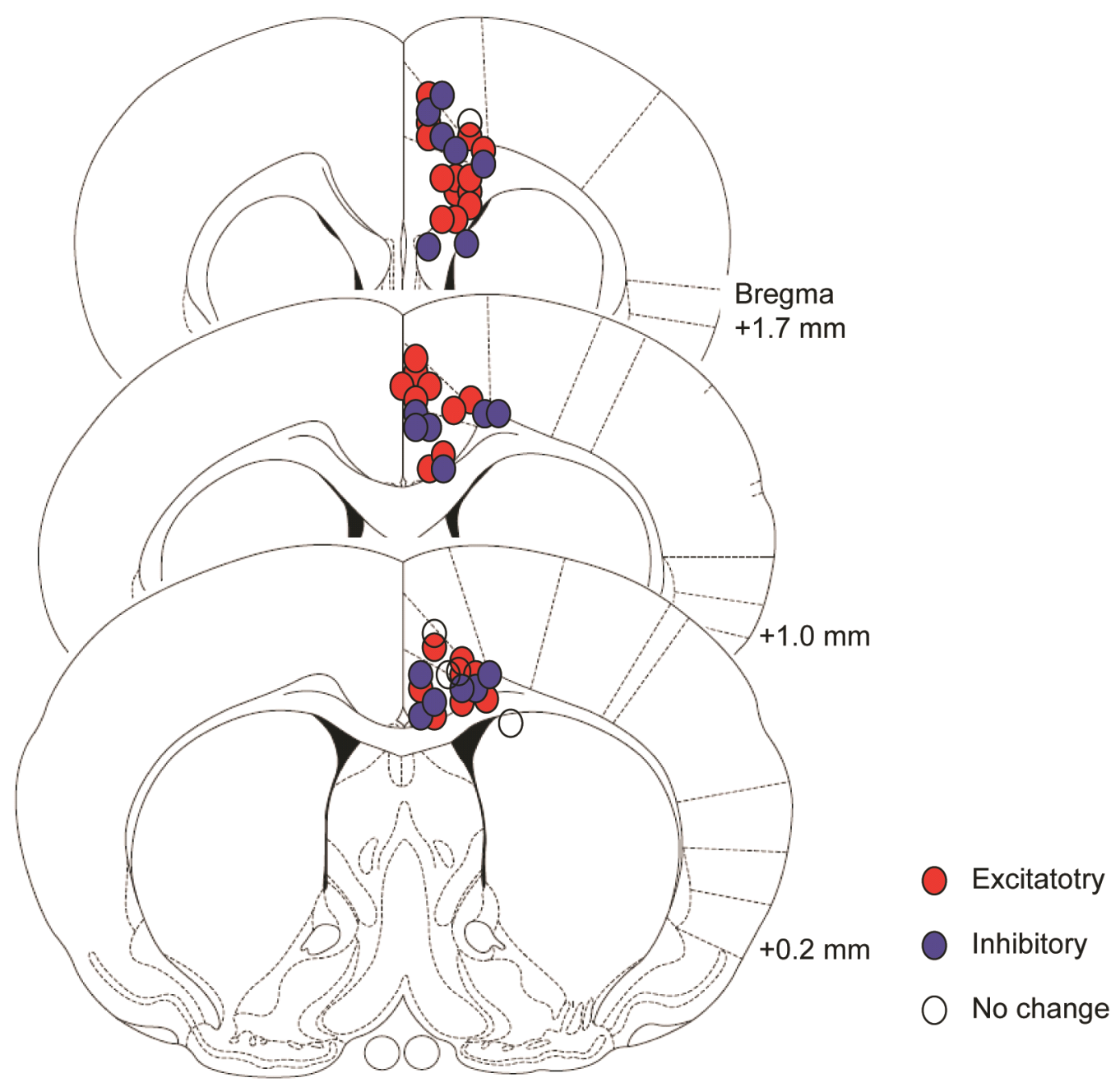

